\title{
Solar Flare Photons and Energetic Particles in Space
}

\author{
E. W. Cliver \\ Air Force Research Laboratory, Hanscom AFB, MA 01731-3010
}

\begin{abstract}
I review the evolution of research on solar energetic particle events, beginning with Forbush's report of the ground level event of 1946, through the most recent observations of the Advanced Composition Explorer (ACE). The emphasis is on research that attempted to link solar flare electromagnetic emissions with the solar energetic particles (SEPs) observed in space following flares. The evolution of thought on this topic is traced from the initial paradigm in which SEPs were accelerated at the flare site (a $\delta$-function in space and time) to the current two-class picture accommodating both impulsive acceleration at the flare site (small ${ }^{3} \mathrm{He}$-rich events) and prolonged acceleration at extended shocks driven by coronal mass ejections (large proton events). I conclude with some open questions; the most prominent of these concerns the relative contributions of the flare and shock acceleration processes to "mixed" or hybrid SEP events in which the distinguishing characteristics of the impulsive and gradual classes are blended.
\end{abstract}

\section{INTRODUCTION}

Within the last 15 years our views of particle acceleration in association with solar flares have undergone a remarkable revision and codification (1). In this review, I trace the steps that led to the current picture. The emphasis will be on observations of solar flare electromagnetic emissions and the solar energetic particles (SEPs) that are observed in space following flares and coronal mass ejections (CMEs). This emphasis on observations reflects my bias but, more importantly, it indicates that ours is a field driven by data. New instruments, such as those on the Advanced Composition Explorer (ACE), inevitably lead to revisions of earlier ideas based on less sophisticated measurements. ACE has already challenged and refined the existing picture. Knowing how our present understanding developed can serve as a guideline to accommodate additional surprises that are certain to come as more events are recorded.

In this paper, I first review the origins of this field beginning with the 25 July 1946 ground level event and continuing through the association of coronal mass ejections (CMEs) and SEPs based on Skylab coronagraph data. The next section of the paper addresses correlations/associations between the intensities of various flare emissions and the sizes of SEP events and the section following that recounts the remarkable flurry of research during the decade from 1984-1993 that established the two-class paradigm. I conclude with a list of open questions for further investigation.

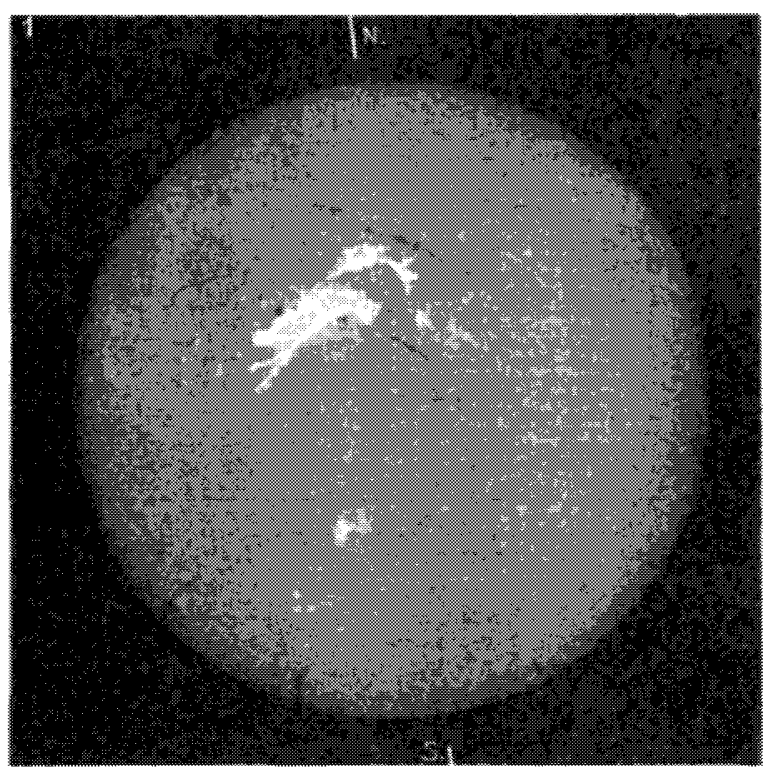

FIGURE 1. Meudon Observatory $\mathrm{H} \alpha$ image of the solar flare associated with the ground level event of 25 July 1946 . From (3), with kind permission of Société Astronomique de France.

\section{ORIGINS}

The history of this field began with the report by Forbush (2) of a sudden transient increase in the counting rate of his ionization chamber on 25 July 1946 in association with a large solar flare. The flare, observed in $\mathrm{H} \alpha$ 


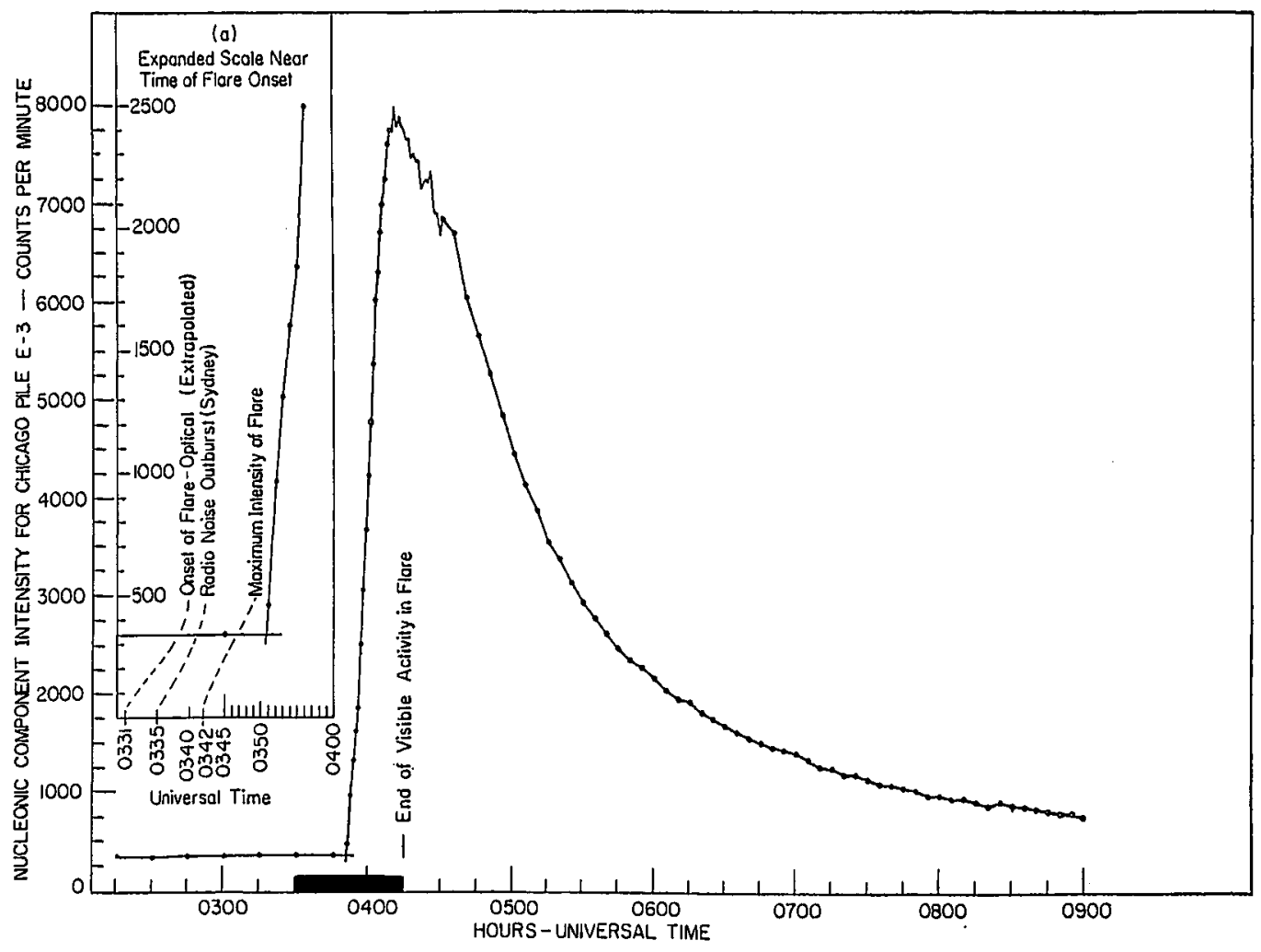

FIGURE 2. Chicago neutron monitor record of the ground level event of 23 February 1956 (adapted from 5).

at Meudon, is shown in Figure 1 (3). Lange and Forbush (4) had earlier observed two similar counting rate increases, occurring in association with solar activity in early 1942, but Forbush did not draw the connection between the earlier increases and the simultaneous flaring until he observed the 1946 event.

The first SEP event to be analyzed in detail was the famous ground level event of 23 February 1956 that was observed by the Simpson-developed neutron monitors at Chicago and five other sites. Meyer, Parker, and Simpson (5) drew attention to the short duration ( $\sim 45$ minutes) of the flare, marked with a heavy line in Figure 2, and the long duration ( $\sim 15$ hours) of the particle event. They concluded that a short-lived acceleration was followed by diffusive propagation. Thus the initial paradigm for particle acceleration in association with solar flares was that all particles were rapidly accelerated at the flare site. SEP acceleration could be modeled as a $\delta$-function injection in space and time (Figure 3), given the relatively small angular extent and short time span of the flare. Actually Figure 3 is anachronistic because the Parker spiral had not yet been hypothesized in 1956 .
As a result of radio observations of flares, various authors suggested that proton events (observed early on as polar cap absorption events with riometers) might be associated with only certain flares, specifically those with long-duration type IV emission. For example, Kundu and Haddock (6) suggested that intense microwave type IV bursts were a signature of proton acceleration. The most systematic expression of the emerging view that "proton flares" were different from other flares was given by J.P. Wild, S.F. Smerd, and A.A. Weiss in an Annual Reviews paper in 1963 (7). By this time, these Australian radio astronomers had already been observing the Sun at meter wavelengths for over a decade. They separated flares into two characteristic types (or phases) as shown in Figure 4 (8). The vast majority of flares exhibited only the phenomena to the left of the dashed line in the figure. Type III radio bursts, attributed to escaping beams of $\sim 100$ $\mathrm{keV}$ electrons, were the characteristic emission of impulsive flares (or of the impulsive phase of fully-developed flares). Certain flares - generally the largest or most intense events - were accompanied by a second phase of radio emission characterized by slow-drifting type II bursts, 


\section{INITIAL PARADIGM FOR PARTICALE ACCELERATION AT THE SUN}

\section{FLARE: $\delta$ FUNCTION IN SPACE \& TIME}

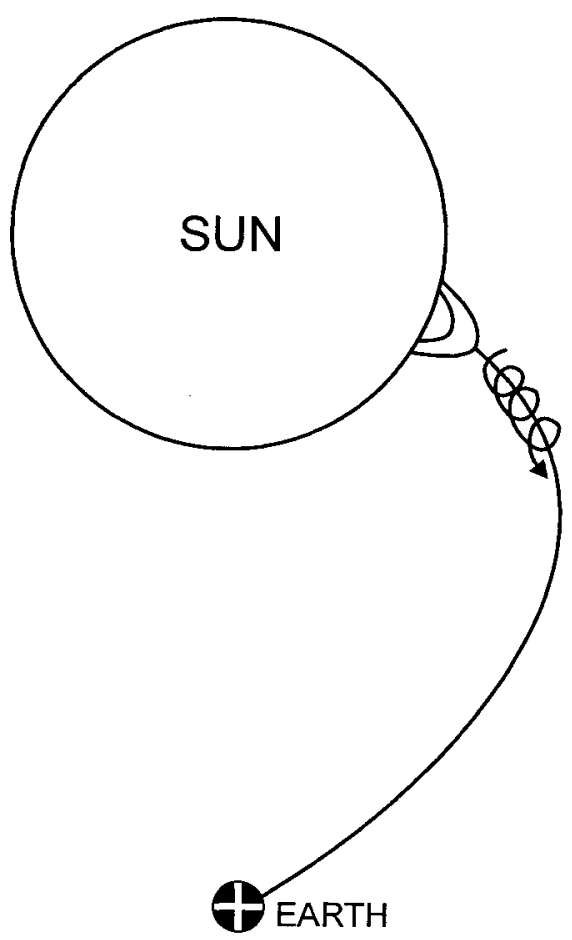

FIGURE 3. The initial paradigm for particle acceleration at the Sun: it was thought that all SEPs were rapidly accelerated at the flare site.

attributed to magnetohydrodynamic shocks moving outward in the corona with speeds $\sim 1000 \mathrm{~km} \mathrm{~s}^{-1}$, as well as by various kinds of type IV emission. Wild, Smerd, and Weiss suggested that protons and high-energy (relativistic) electrons were accelerated in this second phase.

Their suggestion was so close to our current picture that it is worth quoting directly, "Studies of radio emission ... give striking evidence that two separate phases [of particle acceleration] are involved. The first (which is sometimes preceded by a gradual preliminary heating of the flare region) is a succession of bursts of electrons $(\sim 100 \mathrm{keV})$, the acceleration of each being accomplished in a very short time $(\sim 1 \mathrm{sec})$; this requires a catastrophic event, probably involving the conversion of magnetic into kinetic energy ... The acceleration of protons to high energies need not be involved in this phase. The second phase, occurring only in large flares, is initiated directly by the first: the sudden release of energy sets up a magnetohydrodynamic shock wave which travels out through the coronal plasma and creates conditions suitable for Fermi acceleration of protons and electrons to very high ener- gies $(\sim 1 \mathrm{BeV})$." The main differences between this picture and the current paradigm are: (a) the role ascribed to CMEs for shock formation in the modern view $(9,10)$; and (b) the recognition that protons and high-energy electrons are also accelerated in the impulsive phase of energetic flares (11).

Evidence for the "two-phase" picture, as it came to be called, was provided by Lin (12) who reported on the basis of early SEP measurements from space that "pure" electron events were preferentially associated with flares that only exhibited metric type III emission while "mixed" events with protons and relativistic electrons tended to follow flares with type II/IV radio events. Thus, the work of Wild, Smerd, and Weiss and Lin laid the foundation for the current two-class picture of SEP events championed by Reames and colleagues ( 1 , and references therein), although the general acceptance of this view with a host of new details - would take nearly a quarter century following Lin's work.

The last key element of the modern picture was recognized in 1978 when Kahler, Hildner, and Van Hollebeke pointed out an association between CMEs observed by the coronagraph on Skylab and proton events. There was still considerable support for the flare $\delta$-function paradigm (Figure 3) at the time, however, so Kahler et al. hedged their bets on the interpretation. In their conclusion, they suggested that the CME might facilitate observation of protons either by opening field lines so particles accelerated by the flare could escape or, alternatively - the current view - that "there may exist a proton acceleration region above or around the outward moving ejecta far above the flare site."

\section{CORRELATIONS AND ASSOCIATIONS}

Lin and Hudson (13) presented evidence that a large hard X-ray burst was a necessary condition for a major $\sim 10 \mathrm{MeV}$ proton event at Earth. They viewed the intense $\mathrm{X}$-ray emission as evidence for explosive heating of the solar atmosphere, producing a blast wave that drove material out of the Sun's gravitational field and also accelerated the energetic particles. Thus the flare was primary and the ejected mass secondary to the particle acceleration. Kahler (14) injected a note of caution regarding findings such as that of Lin and Hudson that drew associations between phenomena observed in large flares. He posited the existence of a "big flare syndrome" which said, in effect, that big flares have more of everything and that correlations and associations observed in samples of big flares should not be taken as evidence of causality.

How to beat the big flare syndrome? Cliver, Kahler, and McIntosh (15) identified several large proton flares 


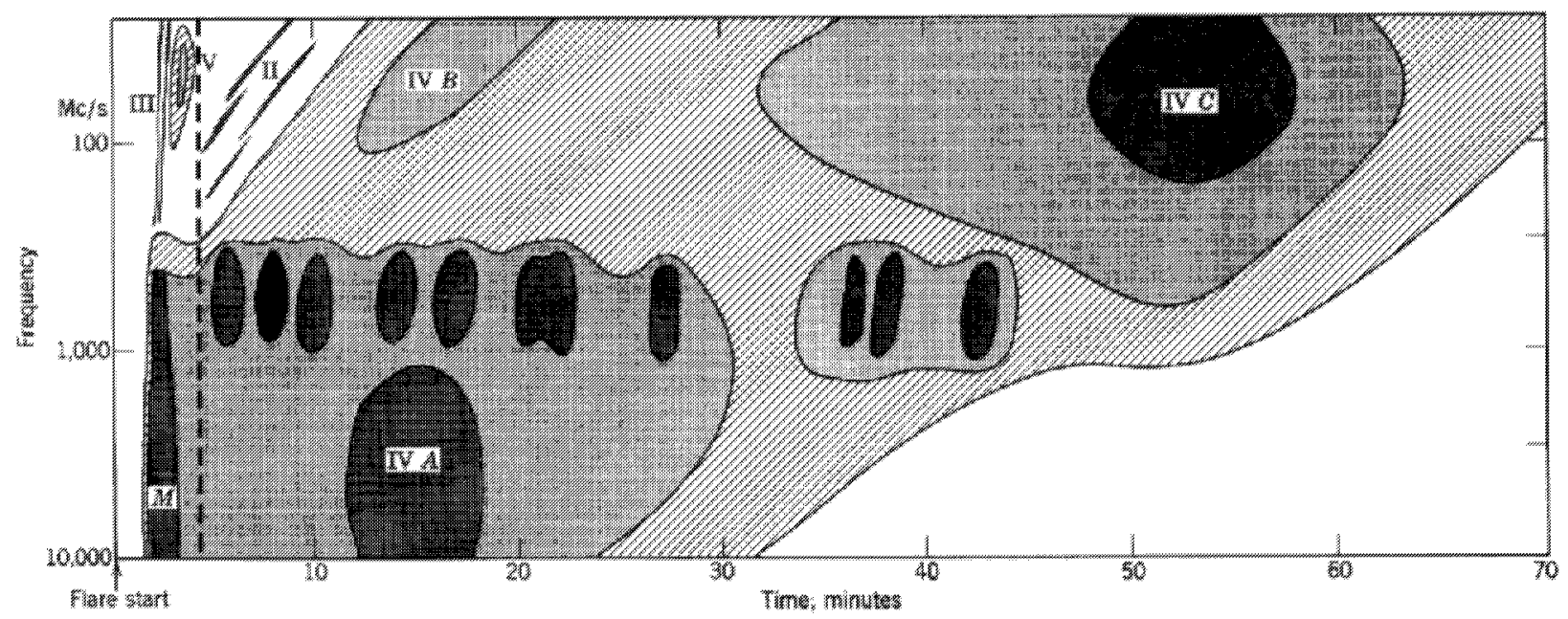

FIGURE 4. Schematic of radio emission for a large "fully-developed" solar flare (8). The dashed line separates the first and second phases. From M. R. Kundu and S. F. Smerd, Inf. Bull. Europ. Solar Radio Obs., 11, 4, Copyright (1) 1962. Reprinted by permission of John Wiley \& Sons, Inc.

that lacked the strong impulsive phases seemingly required by the analysis of Lin and Hudson (13). They determined that these "weak impulsive phase" proton flares (having peak $\sim 9 \mathrm{GHz}$ flux densities $\leq 100$ solar flux units) exhibited clear evidence for mass ejection and shock formation. Cliver et al. concluded their paper as follows: "...the fact that significant proton events can originate in flares without prominent impulsive phases leads us to question the importance of the flash [impulsive] phase in the production of protons observed at $1 \mathrm{AU}$ in even the classic "big flares". For the "big flares" as well as for the weak impulsive phase proton flares, we suspect that the key element leading to shock formation ard the subsequent acceleration of the protons observed at Earth is a magnetically driven mass ejection [italics in the original]." This stood in contrast to the picture of Lin and Hudson in which the impulsive phase energy release was a requirement for $\mathrm{CME}$ formation. Kahler et al. (16) took the study of Cliver et al. (15) one step further when they reported a SEP event that lacked an associated flare. Actually the double-ribbon brightening that accompanied a filament disappearance in this event (Figure 5) can be thought of as a "soft" version of a flare but the absence of the impulsive phase fireworks (e.g., big hard X-ray and microwave bursts) in this event underscored the notion that these emissions are not indicative of the process whereby the protons observed at Earth are accelerated.

Before the launch of the Solar Maximum Mission (SMM) satellite with its Gamma-Ray Spectrometer (GRS) in 1980 (17), proton event intensities at 1 A.U. could only be compared with flare radio and X-ray emissions from electrons back at the Sun. The GRS enabled comparisons of the numbers of protons interacting in the solar atmosphere as deduced from gamma-ray line observations with SEP event intensities in space (e.g., 18 ). The result of a study covering the years 1980-1985(19) is shown in Figure 6, where the peak $10 \mathrm{MeV}$ proton event intensity at $1 \mathrm{~A}$.U. is plotted against the $4-8 \mathrm{MeV}$ prompt gamma-ray line fluence. The poor correlation between

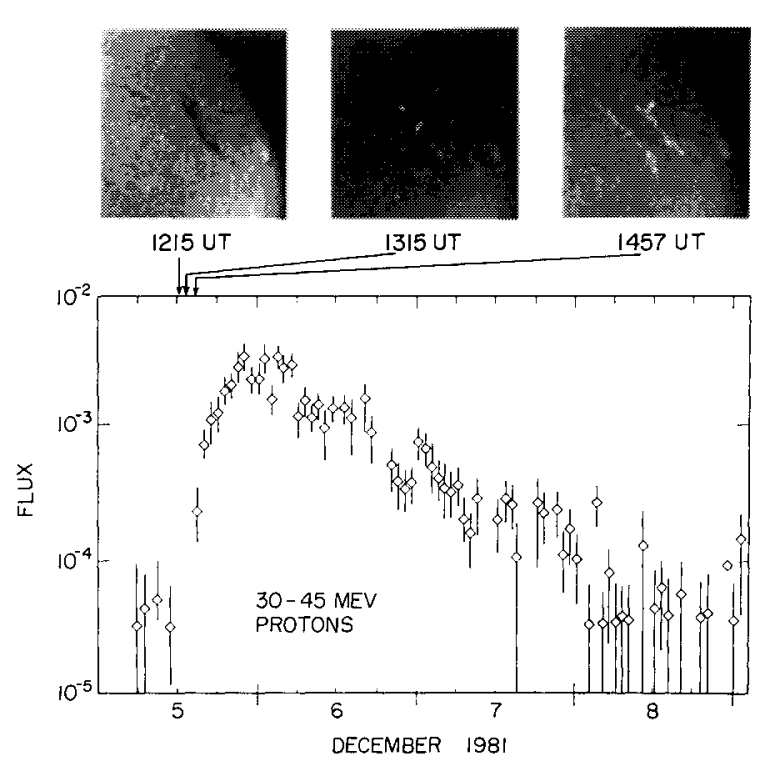

FIGURE 5. The H $\alpha$ erupting filament and SEP event of 5 December 1981 (adapted from 16). 
these parameters results primarily from a class of large SEP events that lacked detectable gamma-ray line emission. These events are in the same class as the weak impulsive phase events of Cliver et al. (15) and include the SEP event associated with a disappearing solar filament reported by Kahler et al. (16); in each case they exhibited evidence for mass ejection and/or shock formation. The poor correlation is consistent with the idea that the protons observed in space are not accelerated in the solar flare.

As a result of these various studies, it has gradually come to be accepted that the largest SEP events originate at a CME-driven coronal/interplanetary shock (Figure 7; 20). As will be discussed later, however, recent ACE observations have raised the possibility that flare accelerated particles may regularly contribute to well-connected SEP events.

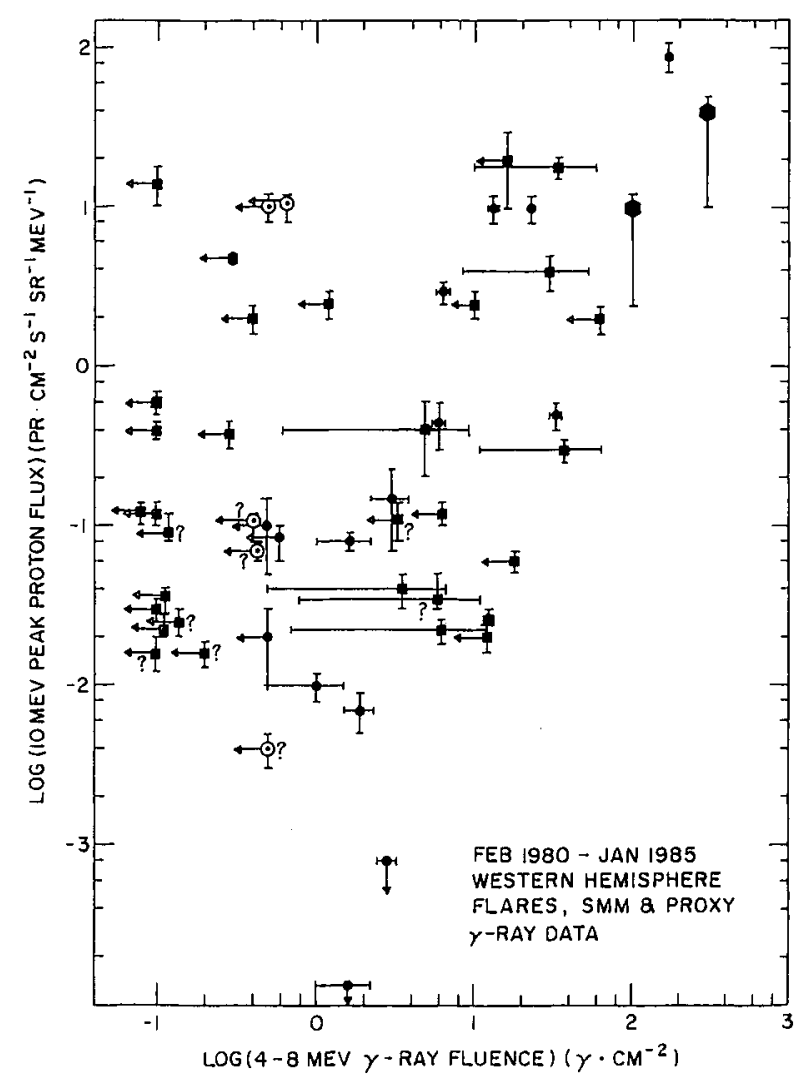

FIGURE 6. Peak $\sim 10 \mathrm{MeV}$ proton flux vs. $4-8 \mathrm{MeV}$ gammaray line fluence for well-connected flares for the first five years of the SMM mission (19).

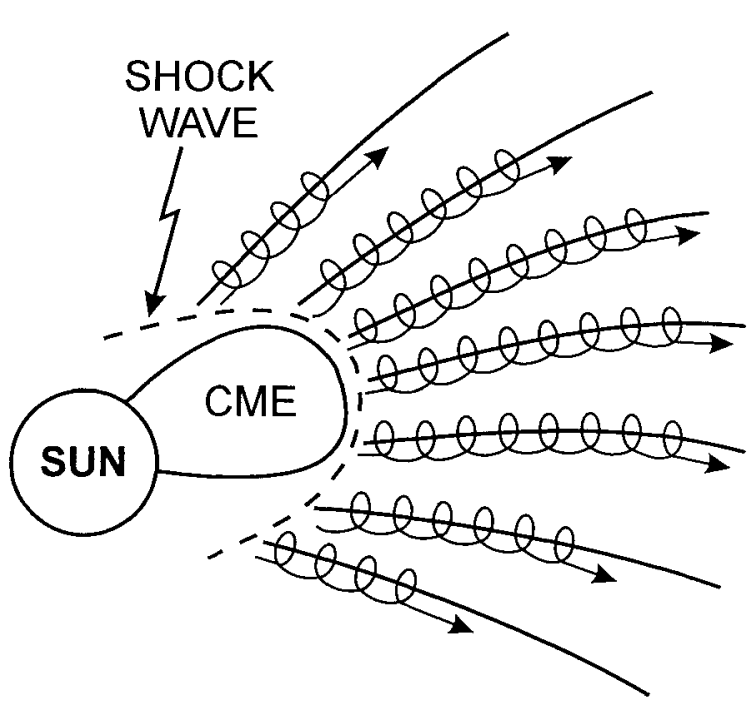

FIGURE 7. Schematic showing SEP acceleration at a CMEdriven coronal/interplanetary shock wave (20).

\section{THE CURRENT PARADIGM}

The current paradigm is the outgrowth of a remarkable period of progress spanning the decade from $\sim 1985$ to $\sim 1995$. The research followed low- and high-energy tracks simultaneously and, in the end, these paths combined to reveal a remarkable synthesis and broad advance in understanding.

\section{Low-Energy SEP Events}

The progress in this area was driven by new instrumentation on the International Sun Earth Explorer (ISEE3) satellite, specifically the ULEZEQ (21) and ULEWAT (21) / VLET (22) instruments, that measured charge states and composition, respectively, of low-energy $(\sim 1$ $\mathrm{MeV} /$ nucleon) ions. The measured charge states were crucial to the adoption of the new paradigm. Klecker et al. (23) were the first to show that ${ }^{3} \mathrm{He}$-rich SEP events (24) and large "normal" SEP events had quite different charge states (Figure 8; see also 25). Hurford et al. (26) found that the ${ }^{3} \mathrm{He}$-rich flares were also enhanced in elements with $\mathrm{Z} \geq 6$. Mason et al. (27) subsequently showed that the composition of the high- $Z$ elements in the large sample of ${ }^{3} \mathrm{He}$-rich SEP events observed by ISEE-3 exhibited a characteristic pattern of enhancement with respect to the elemental abundances observed in large proton events (Figure 9). Thus the charge states and compo- 
sition measurements gave clear evidence for two types of SEP events.

Kahler et al. (28) examined the association of the ${ }^{3} \mathrm{He}-$ rich SEP events with CMEs and coronal shocks and obtained a null result, in contrast to the high degree of association Kahler et al. $(9,10)$ had previously found between large SEP events and CMEs. In a key study, Reames, von Rosenvinge, and Lin (29) used velocity dispersion in one event to associate a ${ }^{3} \mathrm{He}$ enhancement with an increase in low-energy electrons. These studies thus linked ${ }^{3} \mathrm{He}$ enhancements to the flare impulsive phase and separated them from the large flares characterized by CMEs and shock formation. The theory of particle acceleration in the ${ }^{3} \mathrm{He}$-rich events in terms of resonant wave-particle interaction was developed by Temerin and Roth (30) and Miller and Reames (31) (see 1 and 32 for updates).

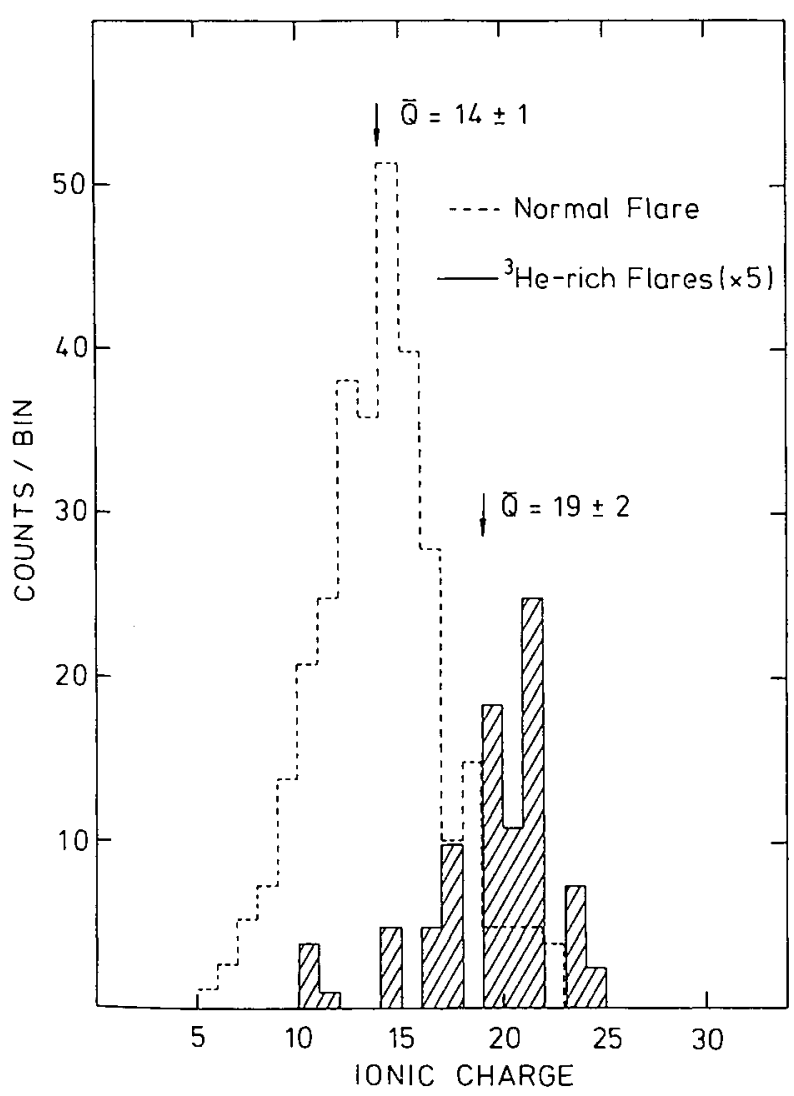

FIGURE 8. Histograms showing the difference in charge state distributions for a large normal SEP event and a sample of ${ }^{3} \mathrm{He}-$ rich SEP events (23).

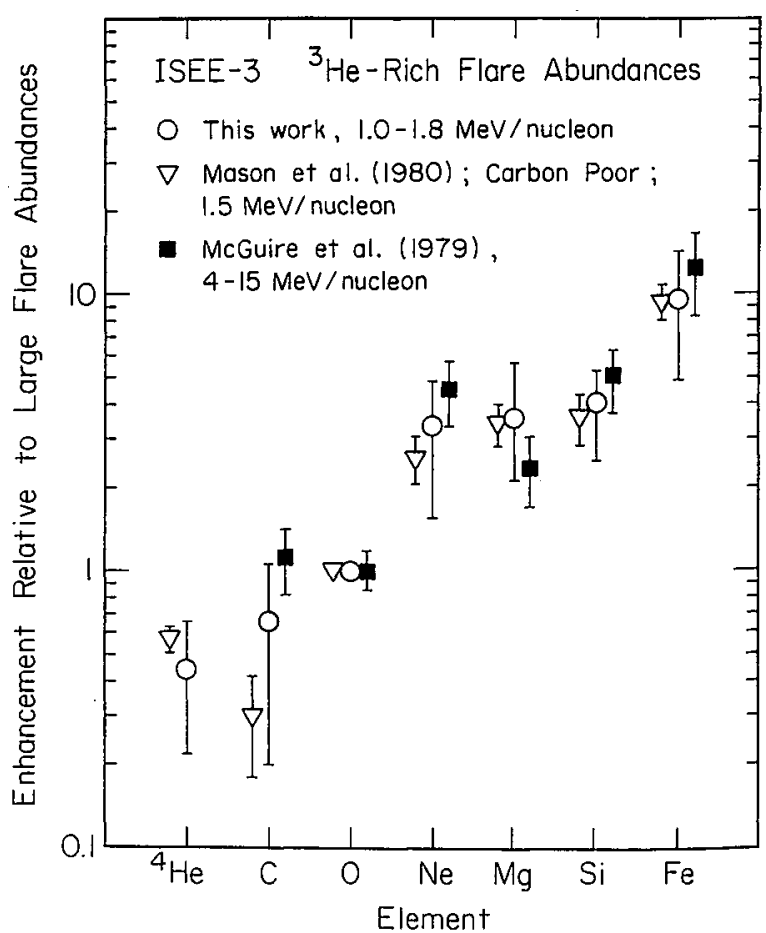

FIGURE 9. Elemental abundances of ${ }^{3} \mathrm{He}$-rich SEP events relative to SEP abundances observed in association with large flares (27).

\section{Observations of SEPs and Photons at High Energies}

The seminal paper for the new paradigm at high energies was that of Evenson et al. (33) who reported the puzzling result that electron-rich SEP events at high energies $(25-45 \mathrm{MeV})$ were preferentially associated with gamma-ray flares, although not all gamma-ray flares were electron-rich. This established a link between certain flares at the Sun and a class of energetic particle events but it also created a mystery. Why only some gammaray flares? The answer was provided in a paper by Cane, McGuire, and von Rosenvinge (34) that represented a reincarnation of sorts of the Wild et al. (7) two-phase picture. Cane et al. showed that impulsive flares with soft X-ray durations $<1$ hour were followed by electronrich SEP events while long-duration flares had "normal" low electron to proton ratios. Only impulsive gammaray flares were associated with electron-rich SEP events. Cane et al. also showed that the gradual flares had broader SEP "cones of emission" than their impulsive counterparts (Figure 10). The analysis of Cane et al. received strong confirmation from a paper by Moses et al. (35) in which it was shown that electron events associated 
TWO CLASSES OF SOLAR PARTICLE EVENTS
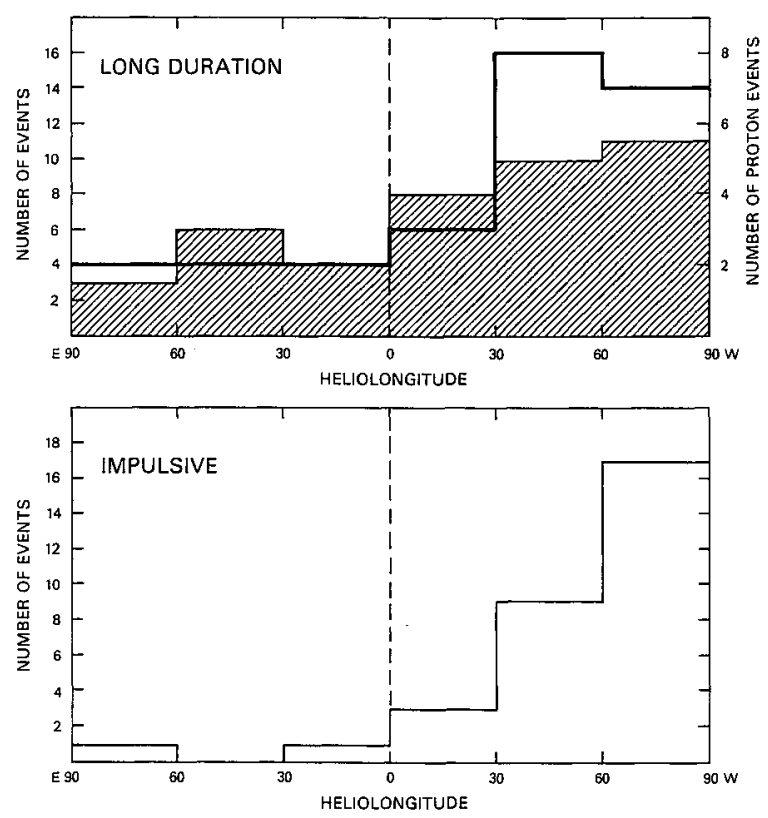

FIGURE 10. Histograms showing the locations of longduration (top) and impulsive (bottom) SEP-associated flares (34). The shaded and unshaded distributions in the upper panel refer to two different samples of large SEP events.

with gradual solar flares had unbroken power-law rigidity spectra while electron events following impulsive flares exhibited a spectral break, with flattening above $\sim 2 \mathrm{MV}$ (Figure 11; cf., 36). At much lower energies, Lin (37) showed that electron spectra for large gradual flares continued to increase without flattening down to the lowest energy measured $(\sim 2 \mathrm{keV})$, indicating a small amount of mass traversal and acceleration high in the corona, while one of two impulsive events from the Cane et al. list that he had data for exhibited a spectral rollover at $\sim 20 \mathrm{keV}$, implying acceleration at heights $\sim 10^{4} \mathrm{~km}$.

In a pioneering paper in 1991, Murphy et al. (38) modeled the gamma-ray measurements of a large gradual flare on 27 April 1981 in terms of an accelerated beam of particles with a heavy element composition similar to that observed for ${ }^{3} \mathrm{He}$-rich SEP events associated with impulsive flares (Figure 12). The basic result of Murphy et al. was confirmed by Share and Murphy (39) by deconvolving the composite spectrum of the 19 largest gamma-ray flares observed by SMM. In addition, Mandzhavidze et al. (40) showed that interacting particles were likely ${ }^{3} \mathrm{He}$ enriched, as expected. These results suggested that while the particles in space (SEPs) came in two basic flavors, the interacting particles in flares came in only one. Ra- maty et al. (41) presented additional evidence for this point of view (cf., 42). For example, Ramaty et al. found that interacting particles had enhanced electron to proton ratios, albeit somewhat higher than those observed for the impulsive events of Cane et al. (34) (cf., 43).

\section{The Two-Class Paradigm}

The new two-class paradigm was articulated in a series of review papers by Reames $(1,44,45,46)$. His synthesis is shown in Table 1, a summation that has become an icon in this field. The cartoons that have been added below

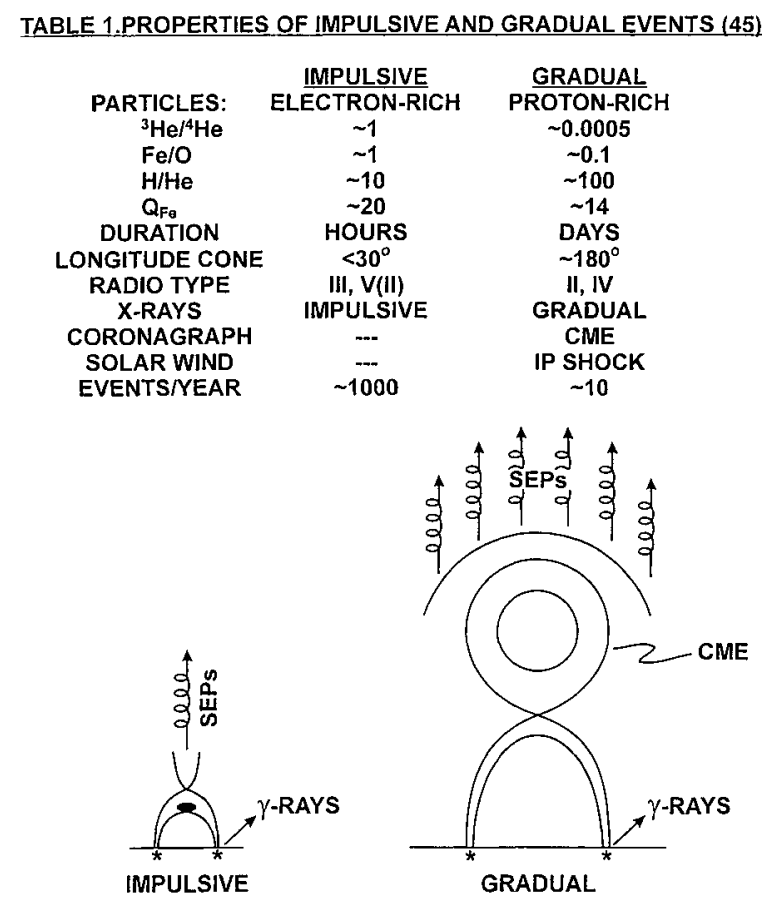

the Table columns show that the new paradigm encompasses the initial $\delta$-function paradigm of particle acceleration in flares (diagram on the left) shown in Figure 3 and the notion that prolonged acceleration could occur at CME-driven shocks. Reames used the Cane et al. (34) two-class picture and the results of the low energy charge state and composition observations to fashion the current picture. In the Reames" synthesis, the "pure" low-energy $(\sim 100 \mathrm{keV})$ electron events of Lin (12) are equated with the more energetic impulsive events of Cane et al. while the results on electron spectra and interacting particles are omitted. Given two basic types of events, one would expect that on occasion hybrids might be observed in which characteristics of both classes are mixed. This can be seen in the diagram on the right in Table 1 where both a flare and a CME-shock can accelerate particles. In fact, for certain well-connected events, there is evidence for 

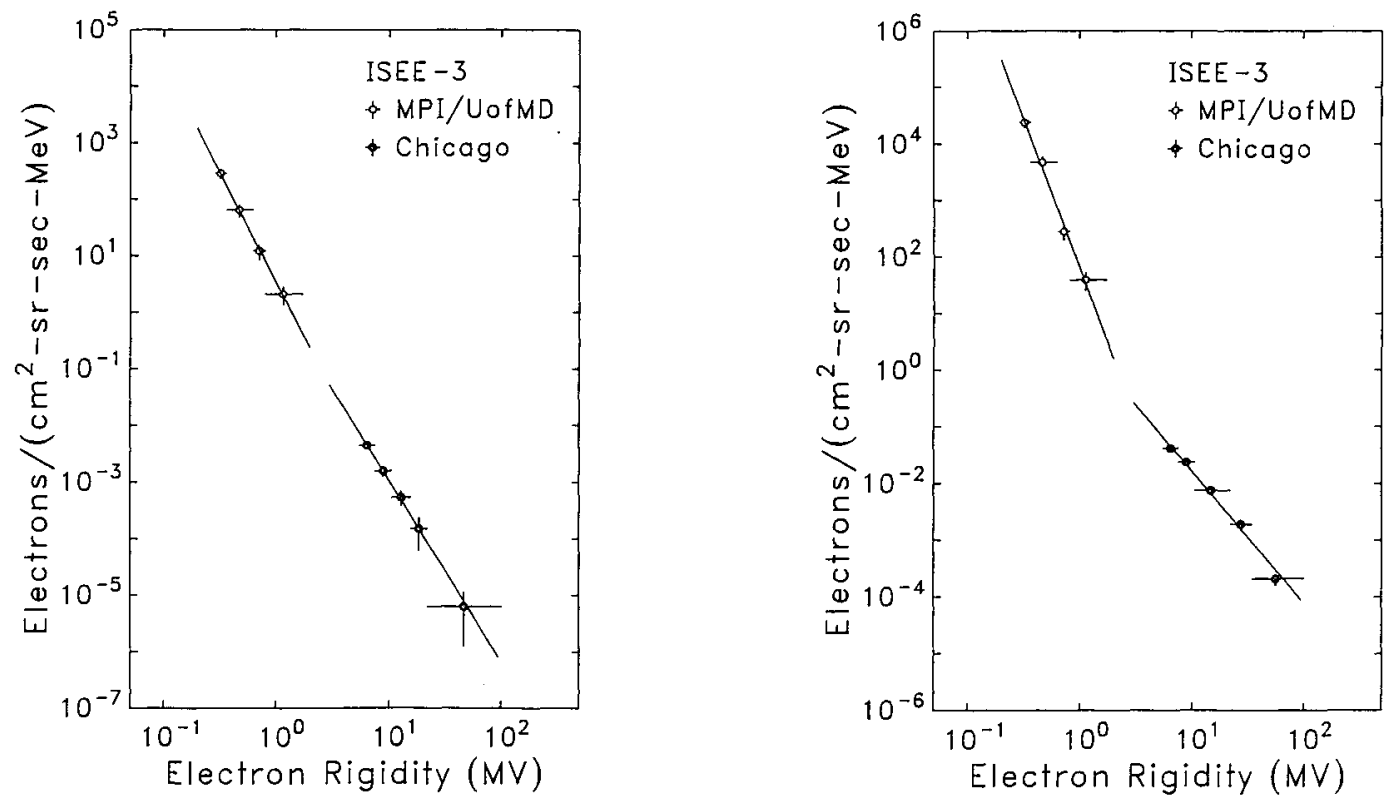

FIGURE 11. Electron rigidity spectra of SEP events associated with gradual (left) and impulsive (right) flares (35).

the evolution of composition from an "impulsive-like" composition early on to a "normal" composition for later stages of the event (e.g., 47, 48). To address the omissions in the standard picture in Table 1, Cliver (42) introduced an expanded table (Table 2) which adds: (1) two additional columns to account for hybrid events (mixedimpulsive and mixed-gradual); (2) four rows at the bottom for interacting particles (common characteristics for both gradual and impulsive flares, i.e., a single flavor); and (3) four additional rows for SEPs to account for electron to proton ratios, the ratio of interacting to interplanetary protons, and high- and low-energy electron spectra.

\section{OPEN QUESTIONS}

It came as somewhat of a surprise that four of the nine largest SEP events observed by ACE through 1998 had compositions and inferred charge states more characteristic of impulsive than gradual events (49; cf., 50). On the basis of the soft X-ray profiles, at least two of the four associated flares would have been classified as impulsive events; a third originated in an active region about two days beyond the west limb, so no time-scale determination is possible. LASCO observations were available for three of the four events and in each case a high speed ( $>1000 \mathrm{~km} \mathrm{~s}^{-1}$ ) CME was observed. The ${ }^{3} \mathrm{He} /{ }^{4} \mathrm{He}$ ratios in these events were enriched by factors of 10-100 above nominal solar wind values $(49,51)$, vs. the nominal factor of 2500 enhancement required for a "pure" impulsive event (Table 1). In the classification scheme of Table 2, these SEP events would be classified as "mixed-impulsive" events based on their soft Xray durations, compositions, charge states, and CME associations. Mason, Mazur, and Dwyer (51) suggest that the ${ }^{3} \mathrm{He}$ enhancements in events like these may be caused by shock acceleration of ${ }^{3} \mathrm{He}$ particles previously accelerated in pure impulsive flares. Reames and his colleagues explain the high $\mathrm{Fe}$ charge states and enhancements of high- $Z$ particles in certain large (and therefore presumably gradual) ACE (and Wind) events in terms of electron stripping (52) and a model of particle acceleration by shocks that takes self-generated waves into account (53), respectively. While they present a compelling case, particularly for the 20 April 1998 SEP event (54), acceptance of their picture implies: (1) that two quite different acceleration/transport processes can produce identical SEP composition; (2) and that the stripping process results in an Fe charge state similar to that observed in impulsive flares.

The need for such coincidences gives one pause and suggests an alternative picture: simply that flare accelerated SEPs account for the observed composition and 
Table 2. Expanded SEP Classification System (42)

\section{Pure Impulsive Mixed-Impulsive Mixed-Gradual Pure Gradual}

\section{Sun}

\begin{tabular}{|c|c|c|c|c|}
\hline Radio Type & III(km III?) & II,III,V & II,III,IV & $\mathrm{IV}(\mathrm{I} ?)$ \\
\hline $\begin{array}{l}\text { SXXR } \\
\text { Duration }\end{array}$ & $<1$ hr(weak) & $<1 \mathrm{hr}$ & $>1 \mathrm{hr}$ & $>1 \mathrm{hr}$ (weak) \\
\hline CME Width & -- & $-20^{\circ}$ & $>45^{\circ}$ & $>45^{\circ}$ \\
\hline Solar Wind & & & & \\
\hline IP Shock & - & - & yes & yes \\
\hline $\begin{array}{l}\frac{\text { SEPS }}{0.5 \mathrm{MeV} \mathrm{e} /} \\
10 \mathrm{MeV} \text { pt }\end{array}$ & - & $>100$ & $<100$ & $<100$ \\
\hline $\mathrm{H} / \mathrm{He}$ & -10 & $>10 ?$ & -100 & $\sim 100$ \\
\hline${ }^{3} \mathrm{He} / \mathrm{He}$ & $\sim 1$ & $<0.1$ & -0.0005 & -0.0005 \\
\hline $\mathrm{Fe} / \mathrm{O}$ & -1 & $<1 ?$ & $\sim 1 \rightarrow \sim 0.1$ & -0.1 \\
\hline $\mathrm{Q}_{\mathrm{pe}}$ & -20 & -20 & $(-20 \rightarrow-14)$ & -14 \\
\hline
\end{tabular}

Electron Spectra

High Energy

Flatten

No Break No Break

Low Energy No Roll-over

Roll-over

No Roll-over No Roll-over

Solar/IP pr

$(-10 \mathrm{MeV})$

$-1-100$

$\sim 0.1-10$

$<0.1$

Longitude Cone $<30^{\circ}$

$-100^{\circ}$

$-200^{\circ}$

$-200^{\circ}$

Interacting Beam

$\mathrm{Fe} / \mathrm{O}$

$(\sim 1)$

$(\sim 1)$

$(<10)$

$(<10)$

-1
$<10$

$?$

$\mathrm{H} / \mathrm{He}$

$>100$

$>100$

$?$

$0.5 \mathrm{MeV}$ e
$10 \mathrm{MeV}$ pr

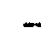

Bremsstrahlung Index $(-0.5 \mathrm{MeV})$

$-3$

?

high Fe charge states in these events. We know from the gamma-ray observations of large flares such as those associated with the large SEP events analyzed by Cohen et al. (49) that particles of impulsive phase composition are produced at the Sun $(38,39,40)$. Moreover, we know that such particles escape from impulsive flares and that only a relatively small fraction of interacting particles needs to escape to produce a detectable SEP event (e.g., 18, 19, 41). It is hard to argue that none of the flare-accelerated particles escape in large gradual flares. The question is 


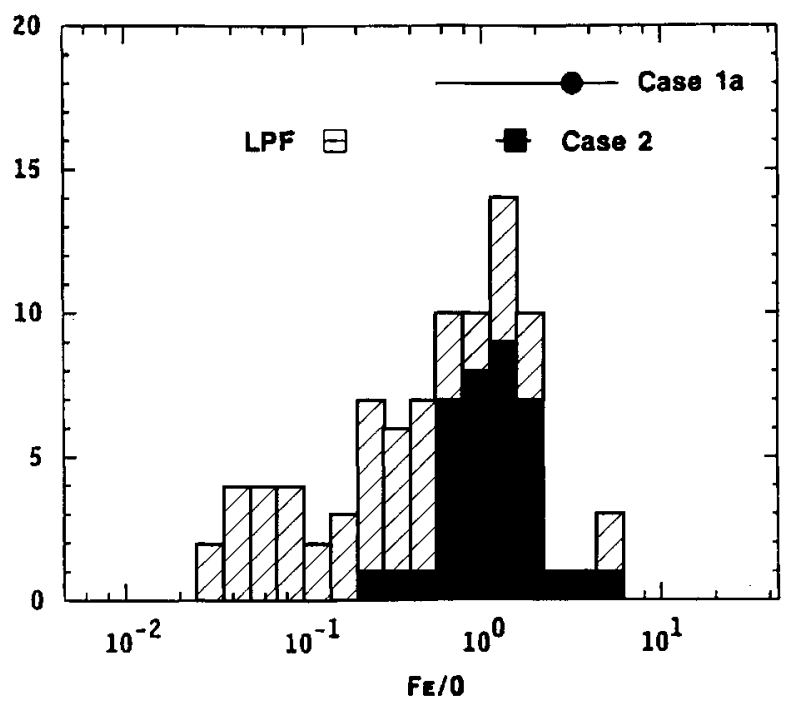

FIGURE 12. Histogram of the distribution of $\mathrm{Fe} / \mathrm{O}$ in SEP events with the ${ }^{3} \mathrm{He}$-rich subset blackened (38). The modeled composition of the accelerated beam based on gamma-ray observations for two separate cases ( 1 a and 2 ) are shown at the top of the plot. The typical large proton flare (LPF) Fe/O ratio of 0.1 is shown by an open square.

- in what proportion in relation to the shock-accelerated particles and at what energies? As pointed out by $\mathrm{Co}-$ hen et al. (49), the four large ACE events they analyzed do not show the strong temporal or spatial variabilities in their compositions that might be expected for the hybrid picture suggested by Reames (48) and Cliver (42) in which flare particles early in an event are followed by shock-accelerated SEPs. As an alternative, Cohen et al. (55) suggest that an energy-dependent superposition of particle populations may be involved.

Other open questions include: (1) the cause of the remarkable separation in classes of electron spectra based on soft X-ray event duration (Figure 11) found by Moses et al. (35); (2) the nature of spatially- (56) and temporally-extended (57) gamma-ray events; and (3) the possible influence of separated coronal and interplanetary shocks on SEP production $(58,59$, cf., 60,61$)$.

\section{CONCLUSION}

From roughly 1985-1995, the research field of solar energetic particle events experienced a paradigm shift from the intial $\delta$-function injection picture (Figure 3) to the current two-class viewpoint (Table 1). Given the general lack of development of the $\delta$-function paradigm, this change can arguably be characterized as a transition from a "pre-paradigmatic state" to one with a well-accepted and detailed framework (1, and references therein). The value of such a framework is clearly revealed in the focus that is brought to the new ACE observations. The first question that is asked is: Do these data fit the paradigm (Table 1)? And, if not, why not? Clearly the new ACE observations have raised interesting challenges to the current paradigm. Ongoing investigations of ACE data, particularly in combination with data from the High Energy Solar Spectroscopic Imager (HESSI; 62) scheduled to fly in July 2000 , promise to provide further insight to a field that has witnessed rapid progress in the last 15 years.

\section{ACKNOWLEDGMENTS}

I thank Dick Mewaldt and Alan Cummings for organizing a stimulating and productive conference and Christina Cohen and Allan Tylka for useful comments.

\section{REFERENCES}

1. Reames, D.V., Space Science Rev., 90, 413 (1999).

2. Forbush, S.E., Phys. Rev., 70, 771 (1946).

3. Servajean, R., and Olivieri, G., Astronomie, 60, 215 (1946).

4. Lange, I., and Forbush, S.E., Terr. Mag., 47, 331 (1942).

5. Meyer, P., Parker, E.N., and Simpson, J.A., Phys. Rev., 104, 768 (1956).

6. Kundu, M.R., and Haddock, F.T., Nature, 186, 610 (1960).

7. Wild, J.P., Smerd, S.F., and Weiss, A.A., Ann. Rev. Astron. Astrophys., 1, 291 (1963).

8. Kundu, M.R., and Smerd, S.F., Inf. Bull. Europ. Solar Radio Obs., 11, 4 (1962).

9. Kahler, S.W., Hildner, E., and Van Hollebeke, M.A.I., Solar Phys., 57, 429 (1978).

10. Kahler, S.W., et al., J. Geophys. Res., 89, 9683 (1984).

11. Forrest, D.J., and Chupp, E.L., Nature, 305, 291 (1983).

12. Lin, R.P., Solar Phys., 12, 266 (1970).

13. Lin, R.P., and Hudson, H.S., Solar Phys., 153, (1976).

14. Kahler, S.W., J. Geophys. Res., 87, 3439 (1982).

15. Cliver, E.W., Kahler, S.W., and McIntosh, P.S., Astrophys. J., 264, 699 (1983).

16. Kahler, S.W., et al., Astrophys. J., 302, 504 (1986).

17. Forrest, D.J., et al., Solar Phys., 65, 15 (1980).

18. von Rosenvinge, T.T., Ramaty, R., and Reames, D.V., Proc. $17^{\text {th }}$ Int. Cosmic Ray Conf., 3, 28 (1981).

19. Cliver, E.W., et al., Astrophys. J., 343, 953 (1989). 
20. Kahler, S.W., Reames, D.V., and Sheeley, N.R., Jr., Proc. $21^{\text {st }}$ Int. Cosmic Ray Conf., 5, 183 (1990).

21. Hovestadt, D., et al., IEEE Trans. Geos. Electr., GE-16, 166 (1978).

22. von Rosenvinge, T.T., McDonald, F.B., Trainor, J.H., Van Hollebeke, M.A., and Fisk, L.A., IEEE Trans. Geos. Electr., GE-16, 208 (1978).

23. Klecker, B., et al., Astrophys. J., 281, 458 (1984).

24. Hsieh, K.C., and Simpson, J.A., Astrophys. J. (Lett.), 162, L191 (1970).

25. Luhn, A., Klecker, B., Hovestadt, D., and Möbius, E., Astrophys. J., 317, 951 (1987).

26. Hurford, G.J., Mewaldt, R.A., Stone, E.C., and Vogt, R.E., Astrophys. J. (Lett.), 201, L95 (1975).

27. Mason, G.M., Reames, D.V., Klecker, B., Hovestadt, D., and von Rosenvinge,T.T., Astrophys. J., 303, 849 (1986).

28. Kahler, S.W., Reames, D.V., Sheeley, N.R., Jr., Howard, R.A., Koomen, M.J., and Michels, D.J., Astrophys. J., 290, 742 (1985).

29. Reames, D.V., von Rosenvinge, T.T., and Lin, R.P., Astrophys. J., 292, 716 (1985).

30. Temerin, M., and Roth, I., Astrophys J. (Lett.), 391, L105 (1992).

31. Miller, J.A., and Reames, D.V., High Energy Solar Physics, eds., R. Ramaty, N.Mandzhavidze, and X.-M. Hua, (AIP Press: Woodbury, NY) AIP Conf. Proc. \#374, p. 450 (1996).

32. Miller, J.A., et al., J. Geophys. Res., 102, 14631 (1997).

33. Evenson, P., Meyer, P., Yanagita, S., and Forrest, D., Astrophys. J., 283, 43 (1984).

34. Cane, H.V., McGuire, R.E., and von Rosenvinge, T.T., Astrophys. J., 301, 448 (1986).

35. Moses, D., Dröge, W., Meyer, P., and Evenson, P., Astrophys. J., 346, 523 (1989).

36. Evenson, P., Hovestadt, D., Meyer, P., and Forrest, D.J., Proc. $19^{\text {th }}$ Int. Cosmic Ray Conf., 4, 74 (1985).

37. Lin, R.P., Proc. $21^{\text {st }}$ Int. Cosmic Ray Conf., 5, 88 (1990).

38. Murphy, R.J., Ramaty, R., Kozlovsky, B., and Reames, D.V., Astrophys. J., 371, 793 (1991).

39. Share, G.H., and Murphy, R.J., Astrophys. J. (Lett.) (in press) (2000).

40. Mandzhavidze, N., Ramaty, R., Kozlovsky, B., Astrophys. $J ., \mathbf{5 1 8}, 918$ (1999).

41. Ramaty, R., Mandzhavidze, N., Kozlovsky, B., and Skibo, J.G., Adv. Space Res., 13(9), 273 (1993).

42. Cliver, E.W., in High Energy Solar Physics, eds., R. Ramaty, N.Mandzhavidze, and X.-M. Hua, (AIP Press: Woodbury, NY) AIP Conf. Proc. \#374, p. 45 (1996).

43. Kallenrode, M.-B., Cliver, E.W., and Wibberenz, G., Astrophys. J., 391, 370 (1992).

44. Reames, D.V., Astrophys. J. Suppl., 73, 235 (1990).
45. Reames, D.V., Adv. Space Res., 13(9), 331 (1993).

46. Reames, D.V., Rev. Geophys. (Suppl.), 33, (U.S. National Report to the IUGG) p. 585 (1995).

47. Mason, G.M., Gloeckler, G., and Hovestadt, D., Astrophys. $J ., 267,844$ (1983).

48. Reames, D.V., Astrophys. J. (Lett.), 358, L63 (1990).

49. Cohen, C.M.S., et al., Geophys. Res. Lett., 26, 2697 (1999).

50. Mazur, J.E., Mason, G.M., Looper, M.D., Leske, R.A., and Mewaldt, R.A., Geophys. Res. Lett., 26, 173 (1999).

51. Mason, G.M., Mazur, J.E., and Dwyer, J.R., Astrophys. $J$. (Lett.), 525, L133 (1999).

52. Reames, D.V., Ng, C.K., and Tylka, A.J., Geophys. Res. Lett., 26, 3585 (1999).

53. Ng, C.K., Reames, D.V., and Tylka, A.J., Geophys. Res. Lett., 26, 2145 (1999).

54. Tylka, A.J., Reames, D.V., and Ng, C.K., Geophys. Res. Lett., 26, 2141 (1999).

55. Cohen, C.M.S., et al., Geophys. Res. Lett., 26, 149 (1999).

56. Vestrand, W.T., and Forrest, D.J., Astrophys. J. (Lett.), 409, L69 (1993).

57. Kanbach, G.O., et al., Astron. Astrophys. Suppl., 97, 349 (1993).

58. Gopalswamy, N., et al., J. Geophys. Res., 103, 307 (1998).

59. Cliver, E.W., Webb, D.F., and Howard, R.A., Solar Phys., 187, 89 (1999).

60. Torsti, J., Kocharov, L.G., Teittinen, M., and Thompson, B.J., Astrophys. J., 510, 460 (1999).

61. Krucker, S., Larson, D.E., Lin, R.P., and Thompson, B.J., Astrophys. J., 519, 864 (1999).

62. Lin, R.P., and the HESSI Team, SPIE, 3442, 2 (1998). 